\title{
Computation of Transient Electromagnetic Fields Due to Switching in High-Voltage Substations
}

\author{
Bukar Umar Musa, Member, IEEE, Wah Hoon Siew, and Martin D. Judd, Senior Member, IEEE
}

\begin{abstract}
Switching operations of circuit breakers and disconnect switches radiate transient electromagnetic fields within highvoltage substations. The generated fields may interfere and disrupt normal operations of electronic equipment. Hence, the electromagnetic compatibility (EMC) of this electronic equipment has to be considered as early as the design stage of substation planning and operation. Also, microelectronics are being introduced into the substation environment and are located close to the switching devices in the switchyards more than ever before, often referred to as distributed electronics. Hence, there is the need to re-evaluate the substation environment for EMC assessment, accounting for these issues. This paper deals with the computation of transient electromagnetic fields due to switching within a typical high-voltage air-insulated substation (AIS) using the finite-difference time-domain (FDTD) method.
\end{abstract}

Index Terms-Air-insulated substation, electromagnetic compatibility (EMC), electromagnetic fields, finite difference time domain (FDTD), switching transients.

\section{INTRODUCTION}

$\mathbf{S}$ UBSTATION electromagnetic environments can be characterized by either measurement or computation. Measurement of the emissions due to switching involves complex and expensive equipment. The alternative is to develop models to predict the emissions. Substation models can be developed to aid in understanding the measurements and to provide means for estimating electromagnetic interference levels beyond measurement limitations [1]. Once the models have been demonstrated to be robust and accurate they have the potential to greatly simplify the analysis process.

Various substation models have been developed since the introduction of automation and control in power systems in general and substations in particular [2]-[5]. Even with the existence of these models, further investigations as suggested in [1], [6], to determine the effect of different materials and structures that may be found in the substation environment on the transient fields, for example transformers, support structures, grounding mesh and the soil content are required. Initially, the emphases of the transient field predictions are mainly on how the radiated field affects the control rooms as most of the electronic equip-

Manuscript received December 08, 2008; revised June 25, 2009. First published February 22, 2010; current version published March 24, 2010. Paper no. TPWRD-00903-2008.

The authors are with the University of Strathclyde, Electronic and Electrical Engineering Dept., Glasgow G1 1XW, U.K. (e-mail: musa_bu@yahoo.com; w.siew@eee.strath.ac.uk; m.judd@eee.strath.ac.uk).

Color versions of one or more of the figures in this paper are available online at http://ieeexplore.iee.org.

Digital Object Identifier 10.1109/TPWRD.2009.2034008 ments are located there. There have been suggestions that the effect of radiated disturbances from busbars may be insignificant in the control rooms [7]. This is because the busbar is assumed to be a poor radiator; and the distance from the busbars to the control rooms is considered to be significantly great for any radiated fields to reduce to a negligible value before reaching the electronic equipment. However, equipment used in the substations has greatly changed, and become highly sophisticated. Moreover and most importantly, some new electronic equipment that has been introduced into the substations are not necessarily housed in the control rooms anymore; it may be located close to the switching devices or sources of disturbance in the switchyard. This is referred to as "distributed electronics" in the switchyards [8]. For the successful introduction and operation of this new equipment in the substations, it must be immune to the interference in this harsh environment. Hence the there is the need to evaluate the transient electromagnetic emissions in the switchyard, taking into consideration points close to the switching devices. The FDTD method can be used to simulate the above scenario and predict the electromagnetic fields effectively.

For the computation of transient fields in a substation, the transient current propagating along the busbars is needed. Instead of using the actual bus currents, a damped sinusoidal current and a sinusoidal current with varying frequency, which are good representations for transient currents in substations, have been used to calculate the radiated fields in this research. Once the capability of the method for the field calculation is established using the assumed current, the next step is to develop suitable method to compute the actual transient current on the busbars, including the physics of the switching elements in the model. The current thus obtained can be used to calculate the transient fields. First, the results obtained with FDTD code for a simple electromagnetic field transient model will be compared with analytical method in a similar environment. Secondly, simulation results for a simplified busbar section in a typical high-voltage substation will be presented.

\section{ANALYTICAL AND FDTD METHODS OF FiELd CALCULATION}

The computation of transient electromagnetic fields in substations is effectively a two-step procedure [2]:

Step 1) calculation of the transient currents on the conductors due to switching;

Step 2) calculation of the resulting radiated fields near the conductors due to the travelling current.

The electromagnetic fields can be calculated by analytical or numerical methods. The hertzian dipole [4], [9] or the analyt- 


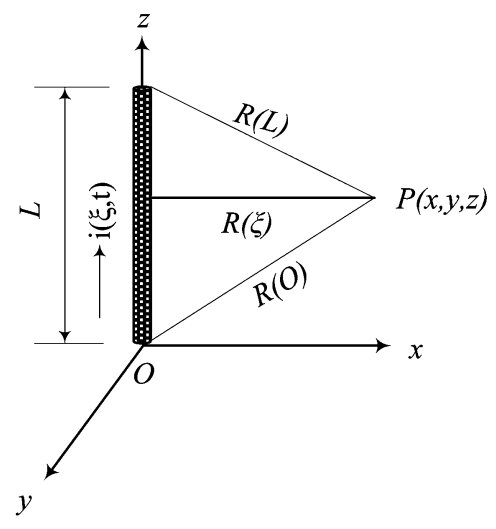

Fig. 1. Vertical filament of current carrying conductor.

ical [10]-[12] method assumes the line or conductor (which is the substation busbar in this case) to be cylindrical. The radius is assumed small in comparison with the minimum wavelength of the transient current so that the current can be regarded as a filament. With reference to Fig. 1 assuming the line is of length $L$, lying along the $z$ axis from the origin of a Cartesian $(x, y, z)$ coordinate system; $P(x, y, z)$ is the coordinate of point of observation, the transient fields can be calculated as follows [10]. Based on the assumptions above, the $x$ and $y$ component of the magnetic vector potential $A$ can be neglected. The electric $(E)$ and the magnetic $(H)$ fields are computed using $A$; for example, the $x$ component of the electric field $\left(E_{x}\right)$ and the $y$ component of the magnetic field $\left(H_{y}\right)$ are given by

$$
\begin{aligned}
& E_{x}(t)=\frac{-x}{4 \pi \varepsilon_{0} c}\left\{\frac{F_{0}(t)}{R^{3}(0)}-\frac{F_{L}(t)}{R^{3}(L)}\right. \\
& \left.\quad-\frac{z}{R(0)} I_{0}(t)+\frac{L-z}{R(0)} I_{L}(t)\right\} \\
& H_{y}(t)=\frac{x}{4 \pi}\left\{I_{o}(t)+I_{L}(t)\right\} \\
& F_{o}(t)=c \int_{0}^{t} i\left(0, \tau-\frac{R(0)}{c}\right) d \tau \\
& F_{L}(t)=c \int_{0}^{t} i\left(L, \tau-\frac{R(L)}{c}\right) d \tau
\end{aligned}
$$

$\begin{array}{ll}I_{0} & \begin{array}{l}\text { current at the lower end of the } \\ \text { conductor; }\end{array} \\ I_{L} & \begin{array}{l}\text { current at the upper end of the } \\ \text { conductor; }\end{array}\end{array}$

$R(0) \quad$ distance from the lower end of the conductor, which is also the origin to the observation point $P$;

$R(L) \quad$ distance from the upper end of the conductor to the observation point $P$;

$c$ speed of light in vacuum;

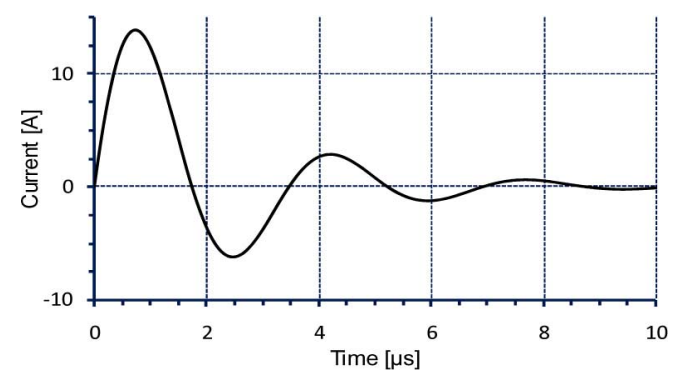

Fig. 2. Damped sinusoidal current.

$$
\begin{array}{ll}
i(0, \tau-(R(0) / c)) & \text { current at } z=0 \text { and time } \\
& \tau-(R(0) / c) ; \\
i(L, \tau-(R(0) / c)) & \text { current at } z=L \text { and time } \\
& \tau-(R(L) / c) ;
\end{array}
$$

$R(\xi) \quad$ distance from an arbitrary point $\xi$ on the conductor to the observation point $P$;

length of the conductor.

From the equations above, it was shown that the fields are functions of the currents at the ends of the conductor [10]. For the comparison of the results obtained using analytical and FDTD methods, the model in Fig. 1 is considered, a single conductor, $30 \mathrm{~m}$ long, vertically oriented as shown. The current applied at the lower end of the conductor, propagating upward is a damped sinusoidal pulse, shown in Fig. 2.

The equation describing the current is: $i(t)=I_{m} e^{-\alpha t} \sin \omega t$ in which $I_{m}=1000 \mathrm{~A}, \alpha=500000, \omega=2 \pi f$, and $f=0.315 \mathrm{MHz}$. With this current, (1) and (2) are solved numerically. The ground is assumed to have constant constitutive parameters that are frequency-independent and is considered to have relative permittivity and permeability values of unity. This model was simulated using both the analytical and FDTD methods to validate the developed method.

The FDTD method is a numerical technique, which models transient electromagnetic signal scattering or radiation from objects of arbitrary shapes. The method relies on discretizing Maxwell's curl equations in both time and space, where all derivatives are approximated by the central difference equation. The computational region, i.e., the region of interest where the solution is sought, is divided into cells with the corresponding electric and magnetic fields being located on the edges and the faces. It usually assumed that all fields in the entire solution region are initially zero. The scattering or radiating objects of interest are defined by specifying the dielectric and magnetic material parameters at each discrete location in the FDTD mesh. The computational region must be large enough to enclose the objects to be analyzed. In addition, suitable boundary conditions on the artificial boundary must be imposed to absorb outgoing waves to simulate the extension of the computational region to infinity [13]. Finally, to ensure the stability of the finite-difference scheme, the time increment $\Delta t$ must satisfy the Courant condition [13], $\Delta t=1 / v \sqrt{1 /(\Delta x)^{2}+1 /(\Delta y)^{2}+1 /(\Delta z)^{2}}$, where $v=\sqrt{\mu \varepsilon}$ is the maximum phase velocity in the model; $\mu$ and $\varepsilon$ are the 
permeability and permittivity of the medium respectively. $\Delta x$, $\Delta y$ and $\Delta z$ are the spatial mesh increments in the $x, y$, and $z$ directions, respectively. To model the above situation, the spatial increments are chosen as $\Delta x=\Delta y=\Delta z=0.5 \mathrm{~m}$ and the discrete time step was set at $95 \%$ of the Courant condition giving $\Delta t=9.141 \times 10^{-10} \mathrm{~s}$. The perfectly matched layer (PML) absorbing boundary condition [14] was implemented for the artificial boundaries to absorb outgoing waves.

\section{A. Thin Wire Representation}

It is a condition of the basic FDTD code that the cell size must be comparable with the smallest structure in the computational space. The radiating elements in the substations are mostly the cables and busbars, which will usually have radius smaller the cell size; it is therefore necessary to include the busbar radius into the physics of the problem. A method of approximating thin wires in the FDTD was required to achieve this. Noda [15] proposed thin wire representation by correcting the adjacent electric and magnetic fields according to its radius and used it for surge simulation. This thin wire representation formulation was adopted in this paper and the conductor radius was set to be $0.025 \mathrm{~m}$. While the use of thin wire representation with a time increment at $99 \%$ of the Courant stability condition has been found to cause numerical instability [16], no instability was experienced at $95 \%$ for the simulations reported here.

\section{B. Reflections at the End of Bus and Ground}

In the analytical method of transient field calculations, reflections at the ends of wire are not considered since it is assumed that the current suddenly "appears" at one end and ultimately "disappears" on the other end of the conductor without reflections. These criteria may not be met in the FDTD formulation adopted here. Of course, there should be reflections at the ends of truncated conductors; nonetheless, the situation is addressed in the FDTD method by placing the upper end of the conductor at boundary of the computational space.

On the reflections due to ground, using the ramp or the Hertzian method, separate techniques must be used to account for the reflections. For example [2], [6] used a method based on Fresnel reflection coefficients and recursive convolution, while [4], [5] used the image method. However, in the FDTD formulation it is a matter of specifying the conductivity, permittivity and permeability at the points, which lie within the ground region. Then the reflection due to ground is automatically incorporated into the computation, which is one of the advantages of the FDTD method.

The ground parameters used were as follows [2], [17]: Conductivity $=0.01 \mathrm{~S} / \mathrm{m}$, Relative permittivity $=10$ and Relative permeability $=1.0$

Having specified all the parameters, the model in Fig. 1 was set up in the FDTD space and simulated. Current was applied at the lower end of the wire as shown in the figure, propagating upward. The transient field calculation assumes matching at both ends. Figs. 3 and 4 show $E_{z}$ and $H_{y}$ components of the electromagnetic fields at a point $P$ located $10 \mathrm{~m}, 20 \mathrm{~m}$ and $50 \mathrm{~m}$ from the centre of the conductor computed by the FDTD as well as the analytical methods. Table I shows the peak-to-peak values of

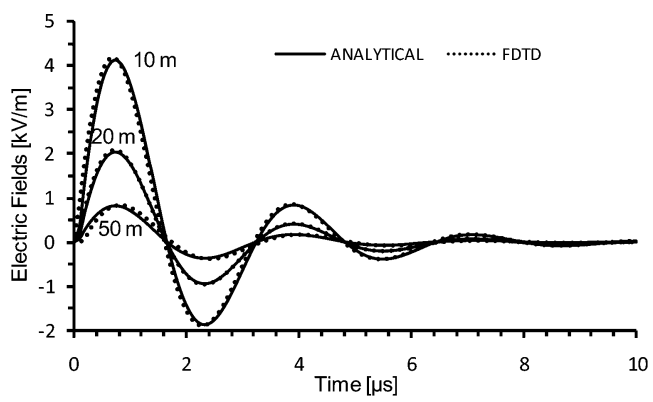

Fig. 3. $E_{z}$ component of electric field produced by the damped sinusoidal pulse for point $P$ located $10 \mathrm{~m}, 20 \mathrm{~m}$, and $50 \mathrm{~m}$ from the centre of conductor.

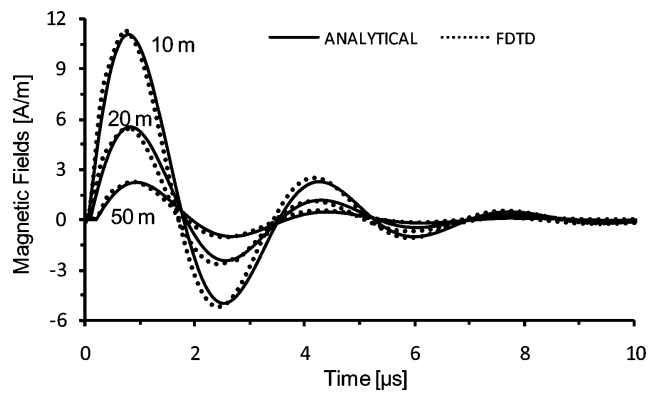

Fig. 4. $H_{y}$ component of magnetic field at a point $P 10 \mathrm{~m}, 20 \mathrm{~m}$ and $50 \mathrm{~m}$ from the centre of conductor produced by the damped sinusoidal pulse.

TABLE I

Peak Values of Electromagnetic Fields $E_{z}$ AND $H_{y}$ Produced by the DAMPED Sinusoidal PUlse at 3 Distances From THE CONDUCTOR

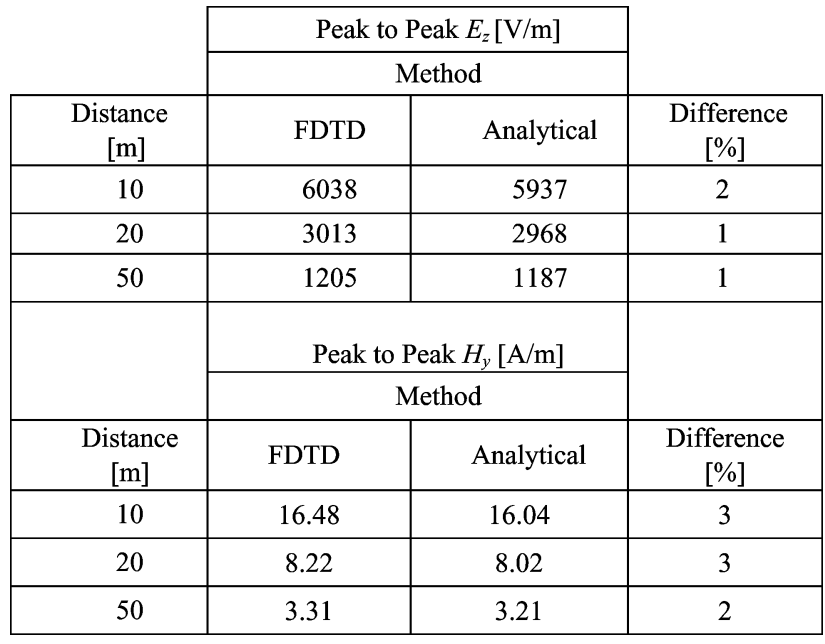

the fields at the same points. It can be seen that there is very good agreement between the two methods in amplitude and phase.

\section{Modelling a Section of High Voltage AIR-INSULATED (AIS) SUBSTATION}

High-voltage busbars in a substation can be modelled by specifying their constitutive parameters within the FDTD grid if the length, radius and position above ground are known. The source location representing the current due to switching can then be specified. To model a more representative section of high-voltage substation for transient field simulation using FDTD method requires specification of the materials within the computational grid, including joints and bends. As the 

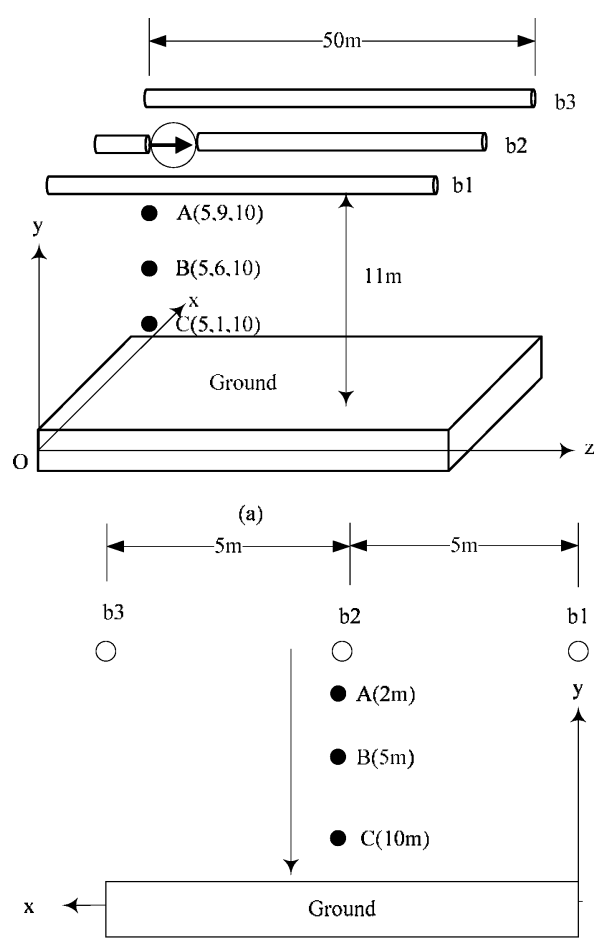

(b)

Fig. 5. Three-phase bus section used in high-voltage substation model. (a) Side view. (b) End view.

complexity of the substation increases, the programming and computation resources also increase. This could even require a mesh generation algorithm to be employed. The simple substation model described here represents a step towards this goal. Typical bus lengths are 20-80 m long and located 5-15 m above ground. Thus, a bus section in a substation consisting of three parallel busbars; $50 \mathrm{~m}$ long, located $11 \mathrm{~m}$ above ground (as shown in Fig. 5) was set up in FDTD space. The ends of the busbars are $2.5 \mathrm{~m}$ away from a 10 - cell perfectly matched layer boundaries. Two different ground conditions were considered for this simulation: imperfect ground and a perfectly conducting ground. The conductor radius used was $0.05 \mathrm{~m}$ in both cases. The imperfect ground properties were chosen as follows: relative permittivity of 10 , conductivity of $0.01 \mathrm{~S} / \mathrm{m}$ and relative permeability of unity. In both cases, a sinusoidal current with varying frequency was used. The frequency was varied from 1 $\mathrm{MHz}$ to $50 \mathrm{MHz}$ in discrete steps. This frequency range was deliberately chosen so that it includes frequencies expected in high-voltage substation transients. Normally switching surges may produce less frequency spectrum than this. However, short duration surges may be expected to occur within this range [1]. This will also enable the analysis of the fields with current of varying frequency.

The current was applied at $10 \mathrm{~m}$ from one end of the middle conductors as shown in Fig. 5 and travels in positive $z$ direction thus simulating a bus section acting as antenna. The peak amplitude of the current was chosen as $1000 \mathrm{~A}$ to emulate a high-voltage bus current transient due to switching. The spatial increments $\Delta x=\Delta y=\Delta z$ were set to $0.5 \mathrm{~m}$ and at $95 \%$ of the Courant condition gives discrete time step as $\Delta t=$
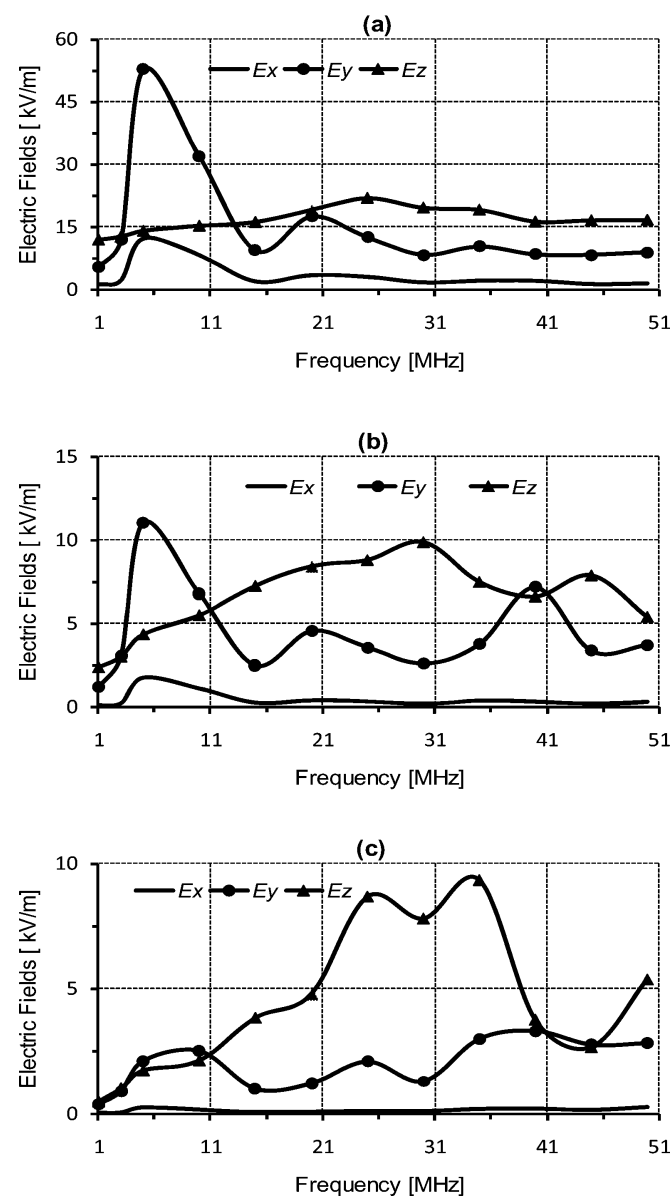

Fig. 6. Variation of electric fields with frequency at three points due to sinusoidal current for imperfect ground conditions. (a) Fields at $A$. (b) Fields at $B$. (c) Fields at $C$.

$9.141 \times 10^{-10} \mathrm{~s}$. The volume of the computational domain was set as $N_{x}=25 \mathrm{~m}, N_{y}=25 \mathrm{~m}, N_{z}=65 \mathrm{~m}$.

$N_{x}, N_{y}$ and $N_{z}$ are the dimensions in the $x, y, z$ directions respectively. The perfectly matched layer absorbing boundary condition was applied as before.

Electric and magnetic fields at three points $A, B$ and $C$ directly under the switch location were calculated and analyzed. Referring to Fig. 5, point $A$ is $2 \mathrm{~m}$ below the busbars, $B$ is $5 \mathrm{~m}$ and $C$ is $10 \mathrm{~m}$ ( $1 \mathrm{~m}$ the above the ground). The coordinates of the points are shown in Fig. 5(a). Fig. 5(b) shows the conductors and the three points in the $x-y$ plane.

\section{A. Effect of Varying Source Frequency}

Fig. 6 shows the $E_{x}, E_{y}$ and $E_{z}$ components of the electric fields at the three points for imperfect ground conditions. $E_{z}$ is the dominant field component except at low frequencies where the $E_{y}$ component is higher. At point $A$, the dominant field is almost constant with increase in frequency. At point $B$, this field increases slightly with increase in frequency up to about $30 \mathrm{MHz}$ and then starts to decrease again.

This may be partly due to the presence of the earth on one hand and the source on the other hand since this point is half way between the two. The $E_{x}$ component of the field is almost negligible here and the behavior is completely dominated by $E_{z}$ as expected, since the radiator is oriented in the $z$ direction. The 

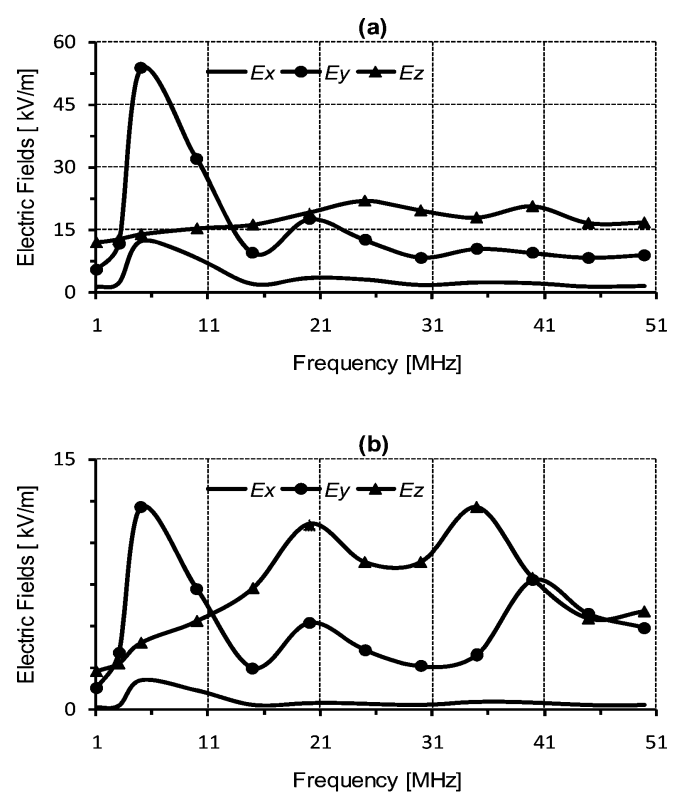

(c)

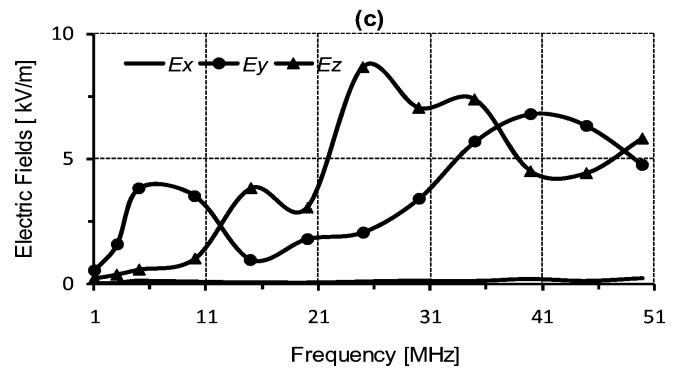

Fig. 7. Variation of electric fields with frequency at three points due to sinusoidal current for perfect ground conditions. (a) Fields at $A$. (b) Fields at $B$. (c) Fields at $C$.

$E_{z}$ field increases with increase in frequency up to $35 \mathrm{MHz}$. At point $C$, both $E_{y}$ and $E_{z}$ increase with frequency up to about $35 \mathrm{MHz}$.

Fig. 7 show the components of the electric field at the same locations $A, B$ and $C$ within the substation for perfect ground conditions.

At point $A$ (far from ground), there is little difference in any component of $E$. At point $B$, the main change is in $E_{z}$. At point $C$, close to ground, both $E_{y}$ and $E_{z}$ are different. $E_{z}$ Shows no noticeable changes.

Fig. 8 shows the components of the magnetic field at the same locations $A, B$ and $C$ within the substation for perfect ground conditions. In all cases $H_{z}$ is negligible compared to the rest of the fields, as would be expected for $z$ directed current. $H_{x}$ is about a factor of 5 higher than $H_{y}$. For points $A$ and $B$ that are further from the ground, fields generally decrease with increase in frequency though the relationship is not linear. Also, the fields have high magnitudes at about $5 \mathrm{MHz}$. For point $C$, which is near the ground, the behaviors of the fields are quite different from the above points. At this point, the field magnitudes increase with increase in frequency up to about $35 \mathrm{MHz}$. This could be due to the proximity to the ground, where induced currents will contribute to the magnetic field. In addition, the fields have high magnitudes at about $5 \mathrm{MHz}$. This could be due
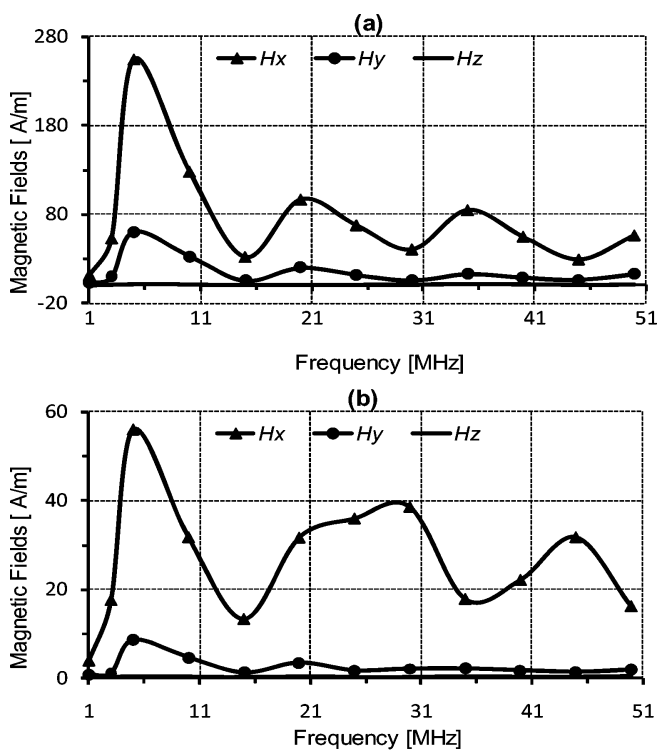

(c)

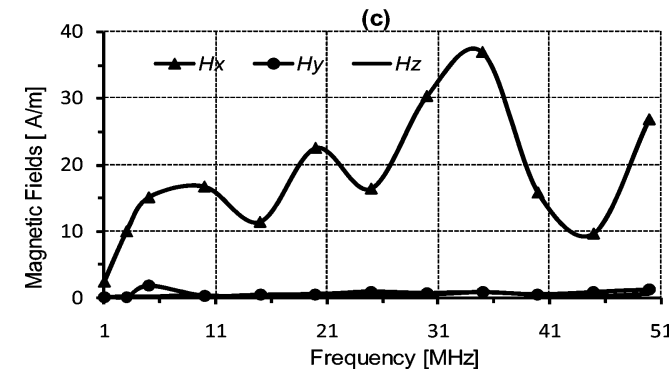

Fig. 8. Variation of magnetic fields with frequency at three points due to sinusoidal current for perfect ground conditions. (a) Fields at $A$. (b) Fields at $B$. (c) Fields at $C$

to resonance effect relating the length of the busbars to the frequency. At this frequency, since the length of the busbar is $50 \mathrm{~m}$, the wave goes into resonance and hence high value of the fields.

Fig. 9 shows the $H_{x}, H_{y}$ and $H_{z}$ components of the magnetic fields at the same locations $A, B$ and $C$ within the substation for imperfect ground conditions.

For points $A$ and $B$, the fields follow the same trend as for the perfectly conducting ground. For point $C$, there is less variation in field with frequency as recorded for the perfect ground. The field variation is almost flat with increase in frequency from 5 MHz up to about $35 \mathrm{MHz}$. Comparing Figs. 8(c) and 9(c), the fields start off at about the same level, but attenuated at high frequencies, due to increasing losses for induced currents in the imperfect ground.

\section{B. Variation of Fields With Distance}

Figs. 10 and 11 summarize the variation of the electric and magnetic field magnitudes respectively with distance for imperfect ground conditions. The field magnitudes have been determined as follows:

$$
\begin{aligned}
& E=\sqrt{E_{x}^{2}+E_{y}^{2}+E_{z}^{2}} \\
& H=\sqrt{H_{x}^{2}+H_{y}^{2}+H_{z}^{2}} .
\end{aligned}
$$



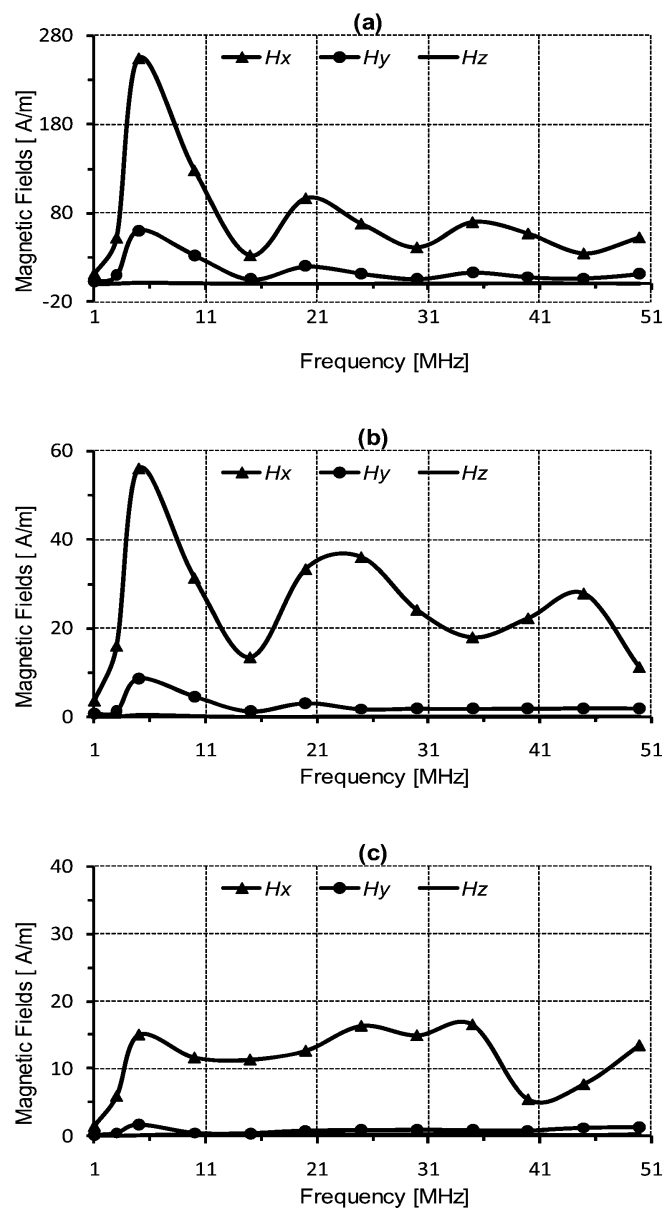

Fig. 9. Variation of magnetic fields with frequency at three points due to sinusoidal current for imperfect ground conditions. (a) Fields at $A$. (b) Fields at $B$. (c) Fields at $C$.

Generally, there is a decrease in magnitudes for both electric and magnetic field strengths with increase in distance from the busbars towards ground. However, at of $35 \mathrm{MHz}$ electric fields are higher at point $C$ than point $B$. In addition, magnetic fields are higher at point $C$ than point $B$ at $50 \mathrm{MHz}$. These can be seen by closer examination of Figs. 10(c) and 11(c).

Figs. 12 and 13 show the variation of the electric and magnetic fields magnitudes respectively with distance for perfect ground conditions. Just as in the imperfect ground situation for both electric and magnetic fields, there is general decrease in field magnitudes with increase in distance from the busbars towards ground. However, magnetic field magnitudes are higher at 35 $\mathrm{MHz}$ and $50 \mathrm{MHz}$ at points $C$ than at point $B$. Also, at $45 \mathrm{MHz}$, magnetic fields are higher at point $B$ than at point $A$. Refer to Fig. 13(c).

\section{Effect of Ground}

With reference to Figs. 10 and 12, it can be seen that the variation of fields with respect to ground conditions depends on the frequency of the current. For example at frequencies of $15 \mathrm{MHz}$, $20 \mathrm{MHz}, 25 \mathrm{MHz}, 30 \mathrm{MHz}$ and $35 \mathrm{MHz}$ (Figs. 10(b)-(c) and 12(b)-(c)) comparison of field magnitudes at point $C$, which is close to the ground, shows that electric fields are the same for both imperfect and perfect grounds. Fields are higher with perfect ground conditions at all other frequencies.

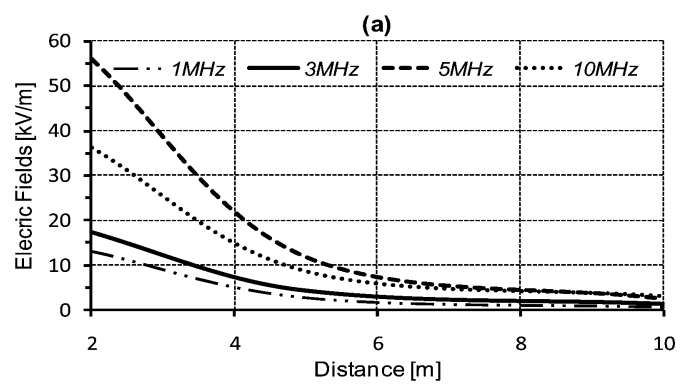

(b)

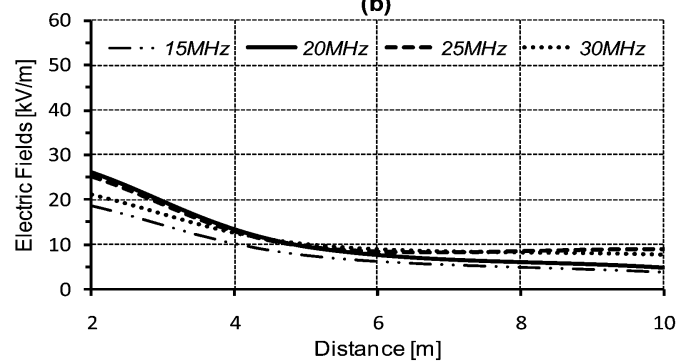

(c)

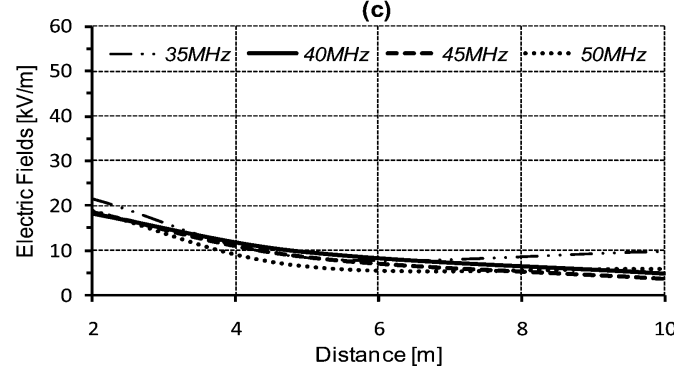

Fig. 10. Variation of electric fields (at twelve frequencies) with distance for imperfect ground.

Referring to Figs. 11 and 13, it can be seen that the variation of magnetic fields with respect to ground conditions also depends on the frequency of the current source.

Magnetic fields are higher with perfect ground conditions than with imperfect ground at $C$ for most of the frequencies. However at frequencies of $5 \mathrm{MHz}, 15 \mathrm{MHz}$ and $25 \mathrm{MHz}$ (Figs. 10(a)-(b) and 12(a)-(b)), the fields are the same for imperfect and perfect grounds. This means that the condition of the ground has a significant influence on the magnetic fields at points in close proximity to the ground, in that a more conductive ground will enhance the transient magnetic fields. Electric fields strengths are rather less affected by ground conditions.

\section{CONCLUSION}

The results presented indicate that FDTD method can be used to model and simulate busbar structures in high-voltage air-insulated substations to predict electromagnetic emissions. Comparison of the FDTD results with analytical solutions gives difference of $3 \%$ or less in all cases. The ability of the code to model the effect of ground conditions has also been demonstrated. It was shown that, in general, perfect ground enhances the radiated fields. Electromagnetic fields generally decrease with distance from the source. Analysis of the simulation results shows that transient electromagnetic field at a point depends on a number of factors. Among these factors are the position relative to the busbar, signal spectrum and the ground conditions. 


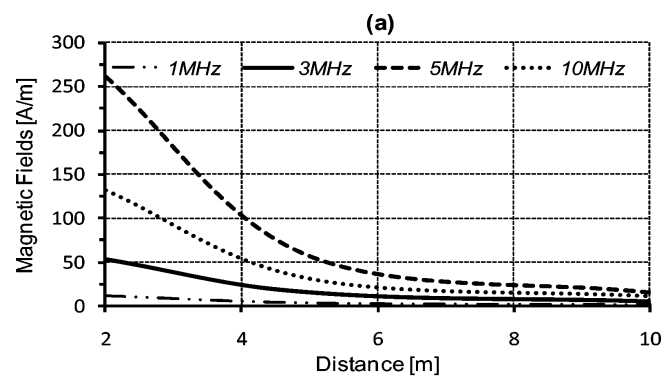

(b)

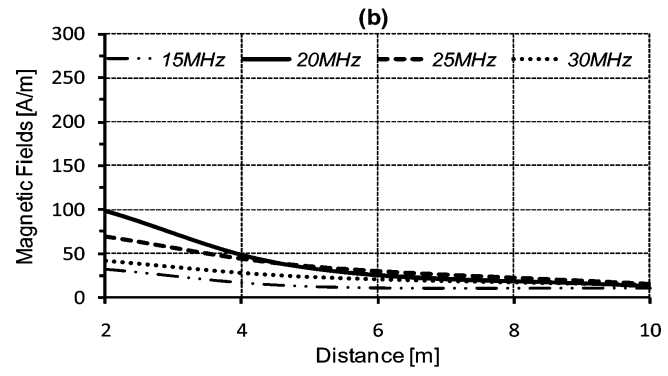

(c)

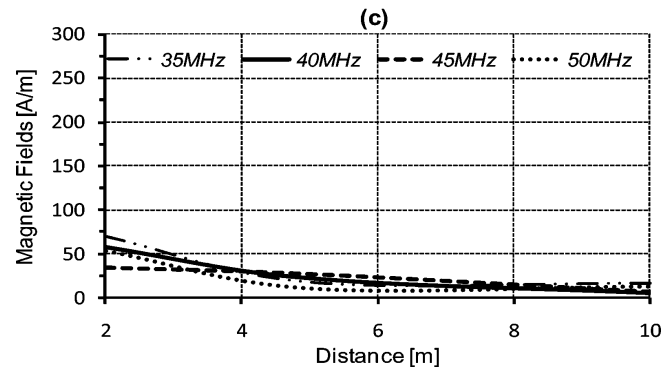

Fig. 11. Variation of magnetic fields (at 12 frequencies) with distance for imperfect ground.

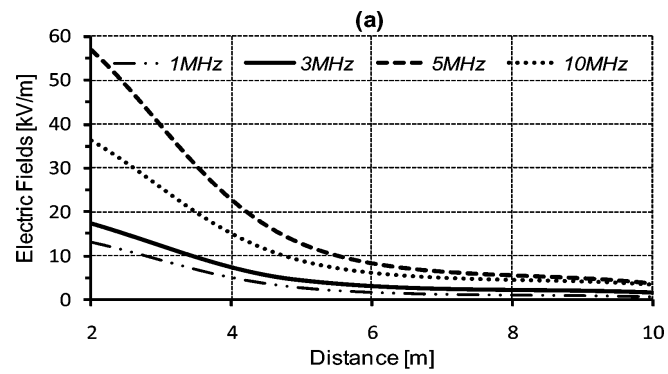

(b)

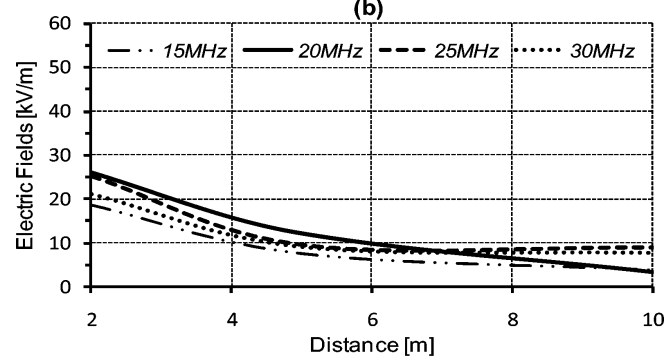

(c)

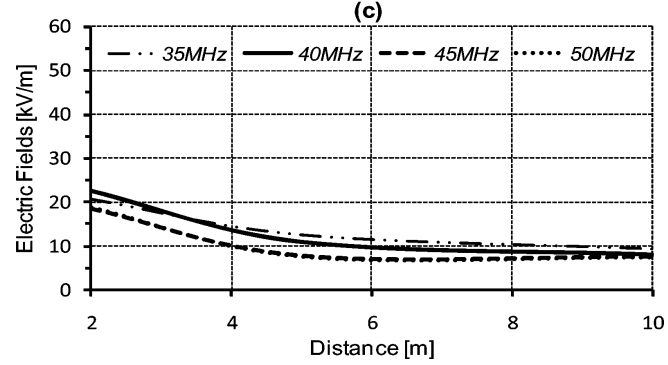

Fig. 12. Variation of electric fields (at twelve frequencies) with distance for perfect ground.

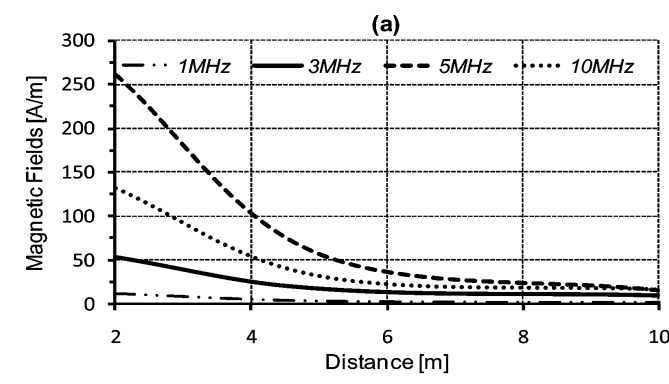

(b)

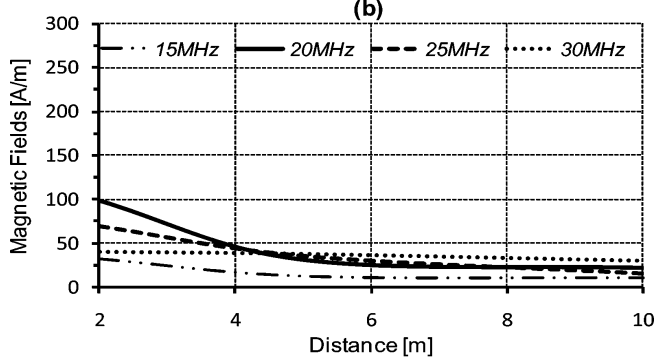

(c)

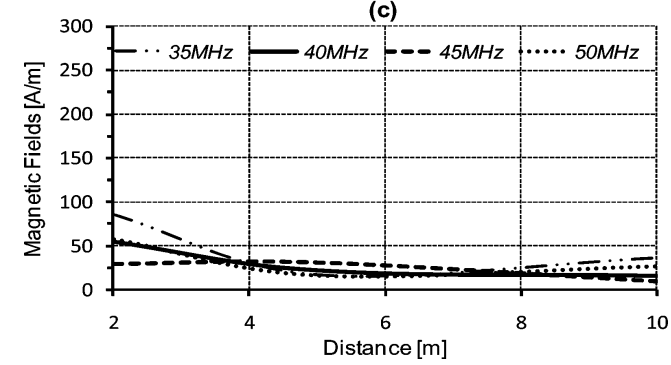

Fig. 13. Variation of magnetic fields (at twelve frequencies) with distance for perfect ground.

Although the model used to test the ability of the simulation is a small section of a substation, this is not a limitation of the code. It would be possible to model a complete high-voltage air-insulated substation, including equipments such as disconnect switch and circuit breakers connected to the busbars, and predict transient electromagnetic fields emissions at any location within the structure. This can be done by specifying the geometry of the various substation components together with their constitutive parameters within the computational space. The predicted transient electromagnetic fields could further be used for electromagnetic compatibility studies and incorporated into the planning and design of substations.

The FDTD model has been used to predict the transient fields in the substation using an analytical waveform transient current due to switching operations. However, an advantage of the developed method is that any type of current waveform can be used as the input source. Extensions to this research could therefore be: 1) to use bus currents obtained from actual substation measurements and 2) develop methods to compute the transient currents produced during switching operations. The second method could finally lead to the development of a comprehensive package that combines the two programs in seamless fashion to run concurrently for predicting electromagnetic fields within a high-voltage substation.

\section{REFERENCES}

[1] C. M. Wiggins, D. E. Thomas, F. S. Nickel, T. M. Salas, and S. E. Wright, "Transient electromagnetic interference in substations," IEEE Trans. Power Del., vol. 9, no. 4, pp. 1869-1878, Oct. 1994. 
[2] D. E. Thomas, C. M. Wiggins, F. S. Nickel, C. D. Ko, and S. E. Wright, "Prediction of electromagnetic field and current transients in power transmission and distribution systems," IEEE Trans. Power Del, vol. 4, no. 1, pp. 744-754, Apr. 1989.

[3] B. Neikhoul, R. Feuillet, and J. C. Sabonnadiere, "Prediction of transient electromagnetic environment in power networks," IEEE Trans. Magn., vol. 30, no. 5, pp. 3745-3748, Sep. 1994.

[4] N. Ari and W. Blumer, "Transient electromagnetic fields due to switching operations in electric power systems," IEEE Trans. Electromagn. Compat, vol. EMC-29, pp. 510-515, Aug. 1987.

[5] E. T. Pereira, D. W. P. Thomas, A. F. Howe, and C. Christopoulos, "Computation of electromagnetic switching transients in a substation," in Proc. Inst. Elect. Eng. Int. Conf. Comp. Electromagnetics., Nov. 1991, pp. 331-334.

[6] A. Orlandi, "On the inclusion of switch arc restrikes and lossy ground in EMI analysis for HV substations," in Proc. 9th Int. Conf. Electromagnetic Compatibility, Conf. Publ. No. 396, Sep. 5-7, 1994, pp. 92-98.

[7] P. H. Pretorius, J. P. Reynders, and A. Fourie, "On radiated EMI generated by disconnector operations in open air high voltage substations," in SAIEE/CIGRE Colloq.: Interference in Power Systems - LF and HF, Midrand, South Africa, Oct. 1999.

[8] C. Imposimato, J. Hoeffel man, A. Eriksson, W. H. Siew, P. H. Pretorius, and P. S. Wong, "EMI characterization of HVAC substations-updated data and influence on immunity assessment," in CIGRE Working Group 36.04(EMC and EMF, General aspects)., 2002.

[9] M. A. Uman, D. K. McLain, and E. P. Krider, "The electromagnetic radiation from a finite antenna," Amer. J. Phys., vol. 43, pp. 33-38, 1975.

[10] R. S. Shi, J. C. Sabonadare, and D. A. Dacherif, "Computation of transient electromagnetic fields radiated by a transmission line: An exact model," IEEE Trans. Magn., vol. 31, no. 4, pp. 2423-2431, Jul. 1995.

[11] B. Neikhoul, R. Feuillet, and J. C. Sabonnadiere, "Prediction of transient electromagnetic environment in power networks," IEEE Trans. Magn., vol. 30, no. 5, pt. 2, pp. 3745-3748, Sep. 1994.

[12] B. Neikhoul and R. Feuillet, "A new method for calculating the transient electromagnetic field radiated by a power transmission line," in Proc. 9th Int. Conf. Electromagnetic Compatibility, 1994, pp. 143-146, IEE Conf. Publ. No 396.

[13] A. Taflove and S. C. Hagness, Computational Electrodynamics the Finite Difference Time Domain Method. Boston, MA: Artech House, 2000.

[14] J. P. Berenger, “A perfectly matched layer," J. Comp. Phy., vol. 114, no. 2, pp. 185-200, Oct. 1994.

[15] T. Noda and S. Yokoyama, "Thin wire representation in finite difference time domain surge simulation," IEEE Trans. Power Del., vol. 17, no. 3, pp. 840-847, Jul. 2002.

[16] Y. Taniguchi, Y. Baba, N. Nagaoka, and A. Ametani, "An improved thin wire representation for FDTD computations," IEEE Trans. Antennas. Propag., vol. 56, no. 10, pp. 3248-3252, Oct. 2008.

[17] A. Maaouni and A. Amri, "Overhead line switching transients," $J$ .Phys.A: Math. Gen., vol. 35, pp. 7125-7135, 2002.

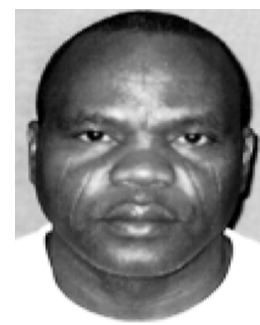

Bukar Umar Musa (M'09) received the B.Eng. degree in Electrical and Electronic (Hons.) at the University of Maiduguri, Maiduguri, Nigeria, in 1994 and the M.Sc. and Ph.D. degrees in electrical engineering from the University of Strathclyde, Glasgow, U.K., in 2003 and 2009, respectively.

In 1995, he joined the Borno State Rural Electrification Board where was actively involved in the design and construction of town distribution networks and intertown connection networks. In 2000 he joined the University of Maiduguri where he is currently a lecturer in the Department of Electrical and Electronic Engineering. His research interests are power system transients, electromagnetic-wave propagation, and computational electromagnetics.

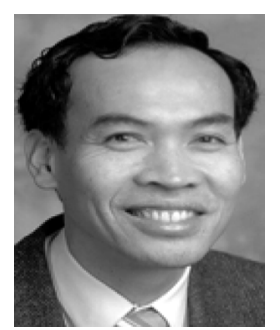

Wah Hoon Siew received the B.Sc. (Hons.) degree in electronic and electrical engineering, the Ph.D. degree in electrical engineering in 1982; and the Master of Business Administration degree from the University of Strathclyde, Glasgow, U.K., in 1978, 1982, and 1985 , respectively

Currently, he is a Reader in the Department of Electronic and Electrical Engineering at the University of Strathclyde, Glasgow, U.K. His research interests include lightning protection to aircraft and grounded structures, electromagnetic compatibility in large installations due to switching and lightning, lightning, EMP and ESD; and cable diagnostics.

Dr. Siew is a Chartered Engineer and a member of the Institution of Engineering and Technology. He is also a member of several CIGRE Working Groups under $\mathrm{C} 4$ and a member of the Technical Panel for the IET Professional Network on electromagnetics.

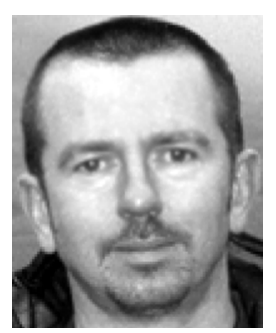

Martin D. Judd (M'02-SM'04) is a Reader with the Institute for Energy and Environment at the University of Strathclyde, Glasgow, U.K., where he manages the High Voltage Diagnostics Laboratory. $\mathrm{He}$ is an expert on electromagnetic theory, propagation, and measurement, and leads a research team specializing in high-frequency diagnostic techniques for high-voltage electrical equipment and systems.

$\mathrm{He}$ is a Chartered Engineer, a member of the IET, and a Fellow of the Higher Education Academy. 